\title{
An exploration into the relationship Irish cancer survivors' have with nutrition post cancer treatment: a qualitative study
}

\author{
N. O'Callaghan ${ }^{1}$, P. Douglas ${ }^{2}$ and L. Keaver ${ }^{1}$ \\ ${ }^{1}$ Department of Health and Nutritional Science, Institute of Technology Sligo, Ash Lane, Sligo, Ireland and \\ ${ }^{2}$ Nutrition Innovation Centre for Food and Health (NICHE), School of Biomedical Sciences, Ulster University, \\ Coleraine, $U K$
}

In Ireland, the number of individuals living with and beyond cancer equates to almost $4 \%$ of the population, with this number projected to increase ${ }^{(1)}$. Little is currently known about how cancer changes perceptions and views on food and nutrition among cancer survivors. Such data gathered from a patient-centered perspective is instrumental for the development of future health interventions in this cohort. The aim of this study was to explore these perceptions and views amongst Irish cancer survivors'.

Participants who were living on the Island of Ireland and had completed active cancer treatment at least six months ago were eligible for this study. Semi-structured focus groups were conducted online via Microsoft Teams in February 2021. The focus groups were audio-recorded and transcribed verbatim. This qualitative data was analysed using Braun and Clarke's updated six-phase process for conducting reflexive thematic analysis ${ }^{(2)}$. Microsoft excel was used to manage the data as the sample size wasn't large enough to require specialised qualitative software. After familiarisation with the data all transcripts were coded using an agreed data coding list. The coded transcripts were analysed for initial themes, which were further reviewed and developed. Finally, themes were refined, defined and named at meetings between the study team (NOC., L.K., and P.D.) until the emergent three themes were agreed upon.

In total, five focus groups were held with 18 cancer survivors. The cohort was predominately female $(\mathrm{n}=16)$ with a mean age of $51.1 \pm 12.5$ years. Three core themes emerged (i) the role of nutrition (ii) cancer as a catalyst for change and (iii) the overall lack of nutritional support in oncology. The emergent themes highlighted how nutrition is perceived to have a variety of roles amongst cancer survivors'. It was seen as a source of empowerment for some, for others as a functional tool to help manage cancer treatment-related side effects and some participants stated how their original perception of nutrition as an important determinant of their health remained. The cohort emphasized how their cancer diagnosis was a prompt for dietary change; predominantly impacting food choices to promote general health. Changes were generally consistent with healthy eating guidelines, although dietary supplements and other non-evidence-based actions were mentioned. Participants reported disappointment that they had not received professional advice about diet and expressed interest in having access to this information.

A cancer diagnosis appears to prompt a re-evaluation of health status among cancer survivors and may potentially prime survivors toward making healthier dietary choices. The views of the participants suggest cancer survivors would welcome guidance from health professionals during and post treatment in relation to their diet and how to nutritionally manage side effects of their cancer treatment.

\section{References}

1. O'Connor M, O'Donovan B, Drummond F, et al. (2019) National Cancer Registry Ireland

2. Braun V \& Clarke V (2021) 'Thematic Analysis' in Analysing Qualitative Data in Psychology, 128-146 